\title{
Electrical cross-talk in four-electrode experiments
}

\section{A digital simulation approach to the example of rotating ring-disk electrodes}

\author{
Soma Vesztergom - Norbert Barankai · Noémi Kovács · Mária Ujvári · \\ Hans Siegenthaler · Peter Broekmann · Győzó G. Láng
}

the date of receipt and acceptance should be inserted later

\begin{abstract}
The subject of this paper is electrical crosstalk, an interference between the current/voltage characteristics of the two working electrodes in four-electrode (generator/ collector) systems. Cross-talk arises in electrochemical cells of finite resistance due to the superposition of the electrical fields of the working electrodes, and often causes difficulties in the interpretation of measurement results. In this paper we present an algorithm for modelling simple generation/collection experiments with a rotating ring-disk electrode (RRDE) immersed into a finite resistance solution of a redox couple. We show that based on the analysis of the Kirchhoff (Laplace) matrix of the simulation mesh, the effect of electrical cross-talk may be accounted for in such experiments. The intensity of cross-talk is found to be heavily influenced by the selection of the reference point for potential measurements; in practice this is the position of the reference electrode or the tip of
\end{abstract}

We dedicate this work to Prof. György Inzelt on the occasion of his $70^{\text {th }}$ birthday and in recognition of his great contribution to electrochemistry.

S. Vesztergom $(\bowtie) \cdot$ N. Kovács · M. Ujvári · G. G. Láng Eötvös Loránd University, Department of Physical Chemistry, Pázmány Péter sétány 1/A, H-1117 Budapest, HUNGARY

Tel.: +36-20-461-2429

E-mail: vesztergom@chem.elte.hu

N. Barankai

MTA-ELTE Research Group in Theoretical Physics,

Pázmány Péter sétány 1/A, H-1117 Budapest, HUNGARY

H. Siegenthaler $\cdot$ P. Broekmann

University of Bern, Department of Chemistry and Biochemistry,

Freiestrasse 3, CH-3001 Bern, SWITZERLAND the Luggin probe. The devised model is validated by means of a simple and demonstrative experiment.

Keywords RRDE - ohmic potential drop · cross-talk effects · digital simulations · Kirchhoff (Laplace) matrix

\section{Introduction}

Four-electrode configurations containing two working electrodes, a single reference and a single counter electrode in the same electrochemical cell were routinely studied over the past half century. A typical example is the rotating ring-disk electrode (RRDE). Developed by Frumkin et al. in 1959 [1], the RRDE was historically the first "generator/collector" system [2] used for studying the intermediates or products formed in electrode processes. If on the generator (disk) electrode some electroactive species are formed as a result of an electrode reaction, these products can be involved in another reaction at the collector (the ring) and can thus be detected [3]. As opposed to many other generator/collector systems (such as inter-digitated arrays [2], where mass transfer between the two electrodes occurs only by the means of diffusion), in case of an RRDE the transfer of species from one electrode to another is also aided by convection, that is a result of rotating the electrode tip.

Recently the use of scanning electrochemical microscopy (SECM) and other related techniques has broadened the application of four-electrode configurations [4]. Using SECM, spatially resolved electrochemical signals can be acquired by measuring the current at an ultramicroelectrode tip as a function of precise tip position over a substrate region of interest [4]. SECM may also be used in a generator/collector mode: 
with an appropriate setting of substrate and tip potentials, species generated at the substrate may subsequently be measured at the tip electrode. An advantage of SECM over the RRDE technique is that it provides means for carrying out generation-collection measurements at controlled generator/collector distances.

Although in most cases of RRDE and SECM experiments steady-state currents are measured, there is a growing interest in the application of transient techniques as well. It was shown for example [5-8] that the simultaneous potentiodynamic perturbation of the generator and collector electrodes can considerably increase the sensitivity of RRDEs. Transient techniques might also be expedient in the case of SECM studies, especially when accurate temporal resolution is to be achieved [9-12]. Measurements in ac-mode were also reported both for RRDE [13-16] and SECM configurations $[17,18]$, and were found to yield useful information on surface-adsorbed intermediates.

Although the application of (bi-)potentiodynamic control in four-electrode systems may indeed have many advantages, some pitfalls have to be identified as well. One of these is a certain interference factor, namely the $I R$-drop related electrical cross-talk that can arise between the current-potential characteristics of the working electrodes in practically any four-electrode configurations with finite (non-zero) resistance. Cross-talk originates from the shared current routes of the two working electrodes and causes an uncompensated potential shift at one electrode, depending on the current flow of the other one. Cross-talk can, in many cases, lead to serious misinterpretations of the current signals measured in 4-electrode systems; this is especially true if transient perturbations resulting in high currents are applied to either one or both of the working electrodes.

Vivier et al. [19] have recently pointed out the existence of a significant electrical cross-talk between the current/potential characteristics of the two working electrodes in SECM setups; a similar effect has long been observed in RRDE experiments [20-23]. In [19-22] four-electrode configurations were analysed in terms of electrical equivalent circuits and it was shown that an electrical cross-talk may be present between the two working electrodes if their current-routes are shared to some extent (e.g., through a common solution resistance). It was also shown [22] that the cross-talk effects become more pronounced in those cases when transient currents are flowing through either one of the working electrodes.

In case of an RRDE the cross-talk effect causes a potential shift at one electrode (ring or disk) depending on the current flow at the other electrode (disk or ring), which can be observed either directly (e.g. under galvanostatic conditions) or in the form of a corresponding current change (under potentiostatic conditions) [23]. The cross-talk may lead to significant errors in the interpretation of experimental curves, and as shown by Shabrang and Bruckenstein [20] - its intensity depends very heavily on the positioning of the reference point for voltage measurement. In an RRDE experiment Shabrang and Bruckenstein [20] observed a minimal cross-talk effect when their Luggin capillary was placed a large distance away from the electrode surface, however a significant cross-talk appeared as the capillary approached the electrode ("Bruckenstein paradox").

In 1980 Dörfel et al. [23] published a very detailed analysis of the problem, where they presented Luggin probe positioning strategies in order to achieve an optimum compromise between "normal $I R$-drops" and cross-talk effects. They also suggested a simple method for the correction of galvanostatically recorded electrode potential vs. time curves in case of exactly known electrode geometries and Luggin probe positions. Dörfel et al., however, could not model the effect of electrical cross-talk on potentiostatically recorded current $v s$. potential curves, noting that this problem "is far more difficult or even impossible" [23].

In this paper we present digital simulations of the RRDE system which can account for the $I R$-drop related cross-talk and its influence on the results of electrochemical collection experiments in case of any arbitrary potentiostatic (or potentiodynamic) perturbation. Results of some test simulations are validated by means of a demonstrative experiment carried out with a simple redox system (a Pt/Pt RRDE immersed into the finite-resistance solution of $\mathrm{K}_{4}\left[\mathrm{Fe}(\mathrm{CN})_{6}\right]$ and $\left.\mathrm{K}_{3}\left[\mathrm{Fe}(\mathrm{CN})_{6}\right]\right)$.

\section{Theory}

Several simulation methods, of varying accuracy, were devised in the past for the numerical description of RRDE experiments [24-27]. A recent algorithm - that also serves as a basis for the approach presented here - was described in details in [27]; the first attempts to include electrical cross-talk into the same simulation algorithm were described in [28].

In this paper we confine our attention to a finiteresistance solution of two electrochemically active species (denoted by Red and Ox) in which a rotating ring-disk electrode tip is immersed. Initially, both Red and $\mathrm{Ox}$ have a uniform concentration distribution in the system, however at the disk and ring surfaces the two 
electroactive species undergo the electrode reaction

$\mathrm{Ox}+\mathrm{e}^{-} \rightleftharpoons$ Red.

We assume that the above reaction is reversible, and thus dictates that the concentrations of the two species in the vicinity of the two electrodes should always fulfil the following equation:

$\frac{c_{\text {Ox near disk or ring }}}{c_{\text {Red near disk or ring }}}=\exp \left[\frac{\left(E_{\text {disk or ring }}-E^{\ominus}\right) F}{R T}\right]$,

that is a special form of the well-known Nernstequation. It should be mentioned here that taking into account irreversible reactions would also be possible within the framework of finite volume simulations by an analytical integration of the Erdey-Grúz-Volmer equation for a given control volume, as described in [27].

We further assume that the concentration changes occurring near the disk and ring surfaces propagate through the system under study as described by the following mass transfer equations:

$$
\begin{gathered}
\frac{\partial c_{\mathrm{Ox}}}{\partial t}=D_{\mathrm{Ox}} \operatorname{div} \operatorname{grad} c_{\mathrm{Ox}}-\boldsymbol{v} \cdot \operatorname{grad} c_{\mathrm{Ox}}, \\
\frac{\partial c_{\mathrm{Red}}}{\partial t}=D_{\mathrm{Red}} \operatorname{div} \operatorname{grad} c_{\mathrm{Red}}-\boldsymbol{v} \cdot \operatorname{grad} c_{\mathrm{Red}},
\end{gathered}
$$

where $D_{\mathrm{Ox}}$ and $D_{\mathrm{Red}}$ are the diffusion coefficients of the reacting species, and $\boldsymbol{v}=\boldsymbol{v}(r, z)$ is the velocity profile of the hydrodynamic flow.

In Eqns. (3.a)-(3.b) we assume that the mass transfer of any electroactive species takes place only by means of diffusion and convection, and that concentration changes due to any other effects (e.g., migration) are negligible. In Eqns. (3.a)-(3.b) we further assume that each diffusing species have a constant diffusion coefficient ( $D_{\mathrm{Ox}}$ and $D_{\mathrm{Red}}$ are independent, for example, from the concentration), and that the velocity profile $v$ is exactly known and is not changing with time at any given $(r, z)$ spatial coordinates. For further details about determining the $v$ hydrodynamic flow profile, the Reader is directed to other sources [27, 29$31]$. In the simulations presented here, we determined $\boldsymbol{v}(r, z)$ by numerically solving the appropriate form of the Navier-Stokes equations describing hydrodynamic motion under a rotating disk as described in [27].

In the simulations we consider a solution of finite electrical resistivity. We assume that the species Red and $\mathrm{Ox}$ are present in concentrations low enough (compared to that of the supporting electrolyte), so that the resistivity of the solution is undisturbed by any changes of $c_{\text {Red }}$ and $c_{\mathrm{Ox}}$, and it is constant over space and time.

For deriving the simulation algorithm we exploit the cylindrical symmetry of the RRDE system. The

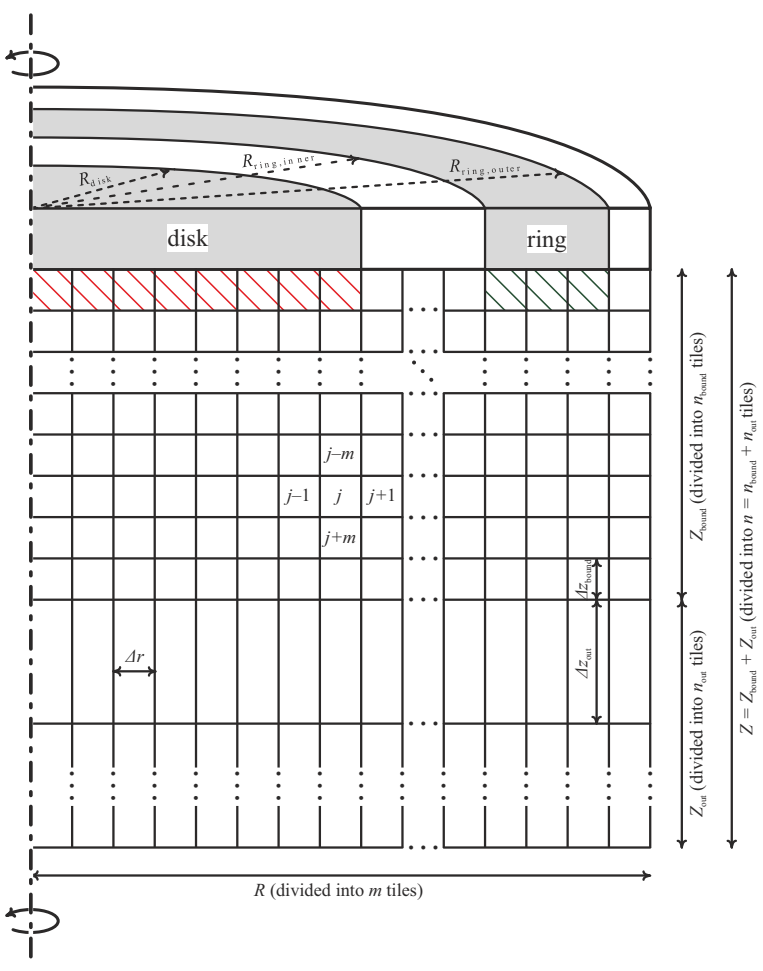

Fig. 1 Two dimensional axi-symmetric mesh used for the spatial discretization of the RRDE system. The space below the RRDE is tiled into small annulus-shaped control volumes. The height of the volumes is smaller in the "boundary layer" close to the electrode surface; a coarser tiling is applied below this layer. Red and green hatching marks volumes which are neighbouring the disk or ring electrodes, respectively. Note the rules of indexing applied: a control volume with index $j$ can have at most four neighbours (with indices $j-1, j+1, j-m$ and $j+m$ ). For an explanation of the used notations, see Tab. 1.

model variables (concentration, velocity, electric potential) are assumed to be a function of two spatial coordinates, the distance $r$ measured from the rotation axis and the distance $z$ measured from the electrode surface. The method of finite volumes is applied: with planes parallel to the electrode surface, the investigated physical space is divided into $n$ layers, and these layers - by a vertical tiling to $m$ segments - are further divided into annulus-shaped control volumes. The symmetry axis of each control volume is the axis of rotation; Fig. 1 shows a sketch of the simulation mesh.

The tiling of the space has to be made smooth enough, so that the model variables can be considered homogeneous inside each control volume within a small time step $\Delta t$ of the simulation. Increasing the smoothness, however, always necessitates the use of smaller $\Delta t$ values (see [27] for setting an appropriate $\Delta t)$ and increases the cost of calculation, which ultimately results in longer computation times. For the simulations presented here, a fine horizontal tiling (re- 


\begin{tabular}{|c|c|}
\hline Parameters & Values \\
\hline \multicolumn{2}{|l|}{ Temporal discretization } \\
\hline Simulation time step $(\Delta t)$ & $3 \mathrm{~ms}$ \\
\hline \multicolumn{2}{|l|}{ Spatial discretization } \\
\hline Full radial spread $(R)$ & $7.5 \mathrm{~mm}$ \\
\hline Number of radial tiles $(m)$ & 40 \\
\hline Radial resolution $(\Delta r)$ & $187.5 \mu \mathrm{m}$ \\
\hline Full vertical spread $\left(Z=Z_{\text {bound }}+Z_{\text {out }}\right)$ & $10.1 \mathrm{~mm}$ \\
\hline Boundary layer thickness $\left(Z_{\text {bound }}\right)$ & $100 \mu \mathrm{m}$ \\
\hline Vert. spread below bound. layer $\left(Z_{\text {out }}\right)$ & $10 \mathrm{~mm}$ \\
\hline Number of vertical tiles & 80 \\
\hline \multicolumn{2}{|l|}{$\left(n=n_{\text {bound }}+n_{\text {out }}\right)$} \\
\hline within the boundary layer ( $\left.n_{\text {bound }}\right)$ & $\begin{array}{l}40 \\
40\end{array}$ \\
\hline \multicolumn{2}{|l|}{ Vertical resolution } \\
\hline within the boundary layer $\left(\Delta z_{\text {bound }}\right)$ & $2.5 \mu \mathrm{m}$ \\
\hline below the boundary layer $\left(\Delta z_{\text {out }}\right)$ & $250 \mu \mathrm{m}$ \\
\hline \multicolumn{2}{|l|}{ RRDE geometry } \\
\hline Disk radius $\left(R_{\text {disk }}\right)$ & $2.5 \mathrm{~mm}$ \\
\hline Disk area $\left(A_{\text {disk }}\right)$ & $0.1964 \mathrm{~cm}^{2}$ \\
\hline Inner ring radius $\left(R_{\text {ring, inner }}\right)$ & $2.679 \mathrm{~mm}$ \\
\hline Outer ring radius $\left(R_{\text {ring, outer }}\right)$ & $2.857 \mathrm{~mm}$ \\
\hline Ring area $\left(A_{\text {ring }}\right)$ & $0.03106 \mathrm{~cm}^{2}$ \\
\hline \multicolumn{2}{|l|}{ Physical parameters } \\
\hline Temperature $(T)$ & $298.15 \mathrm{~K}$ \\
\hline Standard electrode potential $\left(E^{\ominus}\right)$ & $0 \mathrm{~V}$ \\
\hline Initial bulk concentrations $\left(c_{\mathrm{Ox}}=c_{\mathrm{Red}}\right)$ & $10 \mathrm{mmol} \cdot \mathrm{dm}^{-3}$ \\
\hline Diffusion coefficients $\left(D_{\mathrm{Ox}}=D_{\mathrm{Red}}\right)$ & $\begin{array}{l}5 \cdot 10^{-6} \mathrm{~cm}^{2} . \\
\mathrm{s}^{-1}\end{array}$ \\
\hline Kinematic viscosity $(v)$ & $0.01 \mathrm{~cm}^{2} \cdot \mathrm{s}^{-1}$ \\
\hline Conductivity, if finite $(\kappa)$ & $50 \mathrm{mS} \cdot \mathrm{cm}^{-1}$ \\
\hline
\end{tabular}

Table 1 Simulation parameters.

sulting in $n_{\text {bound }}$ layers) was applied for the "boundary region" close to the electrode surface, and a coarser horizontal tiling (resulting in $n_{\text {out }}$ layers) was applied for the space far from the electrode. Numerical values of actual simulation parameters (used to obtain the results presented later on) are listed in Table 1.

In the simulation algorithm the model variables are ordered into vectors of $n \cdot m$ length. In each iteration step we $i$.) realize Nernstian conditions (based on Eq. (2)) in those entries of the concentration vectors which correspond to a control volume neighbouring either one of the two working electrodes; ii.) simulate the effect of mass transfer by solving a discrete version of Eqns. (3.a)-(3.b); and iii.) estimate the uncompensated potential drop affecting the two working electrodes (this depends on the position of the reference point used for voltage measurement).

Details of the calculations are given in Sections 2.1-2.3

\subsection{Charge transfer effects}

Charge transfer only affects those entries $j$ of the $\boldsymbol{c}_{\mathrm{Ox}}$ and $\boldsymbol{c}_{\text {Red }}$ vectors that correspond to control volumes neighbouring either the disk or the ring electrode (see the control volumes marked by a coloured hatching in Fig. 1). In every $i^{\text {th }}$ simulation step, new $c_{\mathrm{Ox}, j}^{(i)}$ and $c_{\mathrm{Red}, j}^{(i)}$ values are determined based on the previous values $\left(c_{\mathrm{Ox}, j}^{(i-1)}\right.$ and $\left.c_{\mathrm{Red}, j}^{(i-1)}\right)$ as

$$
c_{\mathrm{Ox}, j}^{(i)}=\frac{c_{\mathrm{Ox}, j}^{(i-1)}+c_{\mathrm{Red}, j}^{(i-1)}}{1+\exp \left[\frac{\left(E^{\ominus}-\tilde{E}_{\mathrm{disk} \text { or ring }}\right) F}{R T}\right]}
$$

and

$$
c_{\text {Red }, j}^{(i)}=\frac{c_{\text {Ox }, j}^{(i-1)}+c_{\text {Red }, j}^{(i-1)}}{1+\exp \left[\frac{\left(\tilde{E}_{\text {disk or ring }}-E^{\ominus}\right) F}{R T}\right]},
$$

in accordance with the Nernst equation (2). In Eqns (4.a)-(4.b) the $\tilde{E}_{\text {disk or ring }}$ term is the applied potential of the working electrode neighbouring the $j^{\text {th }}$ control volume corrected with the ohmic drop affecting the electrode in question:

$$
\tilde{E}_{\text {disk or ring }}=E_{\text {disk or ring }}-\delta_{I R, \text { disk or ring }} \text {. }
$$

In the $i^{\text {th }}$ step each $j^{\text {th }}$ control volume (with volume $V_{j}$ ) that neighbours either one of the working electrodes gives an

$$
\begin{aligned}
I_{j}^{(i)} & =\frac{F V_{j}\left(c_{\mathrm{Ox}, j}^{(i)}-c_{\mathrm{Ox}, j}^{(i-1)}\right)}{\Delta t} \\
& =\frac{F V_{j} c_{\mathrm{Ox}, j}^{(i-1)}+c_{\mathrm{Red}, j}^{(i-1)}}{\Delta t\left(1+\exp \left[\frac{\left(E^{\ominus}-\tilde{E}_{\mathrm{disk} \text { or ring }}\right) F}{R T}\right]\right)}-\frac{F V_{j} c_{\mathrm{Ox}, j}^{(i-1)}}{\Delta t}
\end{aligned}
$$

contribution to the current of that electrode; by summing up these currents, the values of $I_{\text {disk }}$ and $I_{\text {ring }}$ can be determined.

The values of $\tilde{E}_{\text {disk }}$ and $\tilde{E}_{\text {ring }}$ are optimized in each simulation step by using a Nelder-Mead (downhill simplex [32]) root-finding algorithm. The optimum $\tilde{E}_{\text {disk }}$ and $\tilde{E}_{\text {ring }}$ values, when used in Eqns. (6.a)-(6.b), give rise to $I_{\text {disk }}$ and $I_{\text {ring }}$ currents which in turn estab-

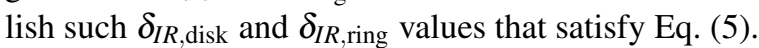
The calculation of $\delta_{I R \text {,disk }}$ and $\delta_{I R \text {,ring from } I_{\text {disk }} \text { and }}$ $I_{\text {ring }}$ is described in details in Section 2.3. 


\subsection{Mass transfer effects}

While the electrode reactions only cause direct concentration changes in those control volumes which are under either one of the working electrode, mass transfer affects all the entries of the $\boldsymbol{c}_{\mathrm{Ox}}$ and $\boldsymbol{c}_{\mathrm{Red}}$ vectors. The general transport equations (3.a)-(3.b) can be rewritten in the form of a matrix-vector equation as

$\boldsymbol{c}_{\text {Ox or Red }}^{(i)}=\left(\mathrm{I}+D_{\text {Ox or Red }} \mathrm{D}-\mathrm{V}\right) \boldsymbol{c}_{\text {Ox or Red }}^{(i-1)}$,

where $\mathrm{I}$ is the $n m \times n m$ identity matrix and the $\mathrm{D}$ diffusor and $\mathrm{V}$ conveyor operators (also $\mathrm{nm} \times \mathrm{nm}$ matrices) are defined as

$\mathrm{D}_{k, \ell}=\left\{\begin{array}{c}\frac{A_{\text {sup }, k} \Delta t}{V_{k} d_{k, \ell}} \\ \frac{A_{\text {int, }, k t} \Delta t}{V_{k} d_{k, \ell}} \\ -\frac{\Delta t}{V_{k}}\left(\frac{A_{\text {sup }, k}}{d_{k, k-m}}+\frac{A_{\text {int }, k}}{d_{k, k-1}}+\frac{A_{\text {ext }, k}}{d_{k, k+1}}+\frac{A_{\text {inf }, k}}{d_{k, k+m}}\right) \\ \frac{A_{\text {ext }, k} \Delta t}{V_{k} d_{k, \ell}} \\ \frac{A_{\text {inf }, k} \Delta t}{V_{k} d_{k, \ell}} \\ 0\end{array}\right.$

if $\ell=k-m$

if $\ell=k-1$

if $\ell=k$

if $\ell=k+1$

if $\ell=k+m$

otherwise

and

$\mathrm{V}_{k, \ell}=\left\{\begin{array}{cl}\frac{v_{r, k} \Delta t}{d_{k, \ell}} & \text { if } \ell=k-1 \\ -\Delta t\left(\frac{v_{r, k}}{d_{k, k-1}}+\frac{v_{z, k}}{d_{k, k+m}}\right) & \text { if } \ell=k \\ \frac{v_{z, k} \Delta t}{d_{k, \ell}} & \text { if } \ell=k+m \\ 0 & \text { otherwise }\end{array}\right.$

In the definitions (8)-(9) $d_{k, \ell}$ denotes the distance of the centres of the $k^{\text {th }}$ and the $\ell^{\text {th }}$ control volumes; $A_{\text {sup }, k}, A_{\text {int }, k}, A_{\text {ext }, k}$ and $A_{\text {inf }, k}$ denote the superior, interior, exterior and inferior bounding surface areas of the $k^{\text {th }}$ control volume with a volume of $V_{k} ; v_{r, k}$ and $v_{z, k}$ denote, respectively, the radial and axial flow velocity components at the center point of the $k^{\text {th }}$ control volume.

Here we note that $\mathrm{D}$ and $\mathrm{V}$ are both large, however sparse banded matrices containing non-zero entries in only five (respectively, three) of their diagonals. Therefore, instead of storing the full matrices we only deal with their non-zero diagonals in the simulation algorithm, which gives a significant increase to the computation speed.

Provided that a smooth enough tiling is used in combination with a small $\Delta t$ value, results of the simulation agree very well with basic theoretical predictions, in accordance with the conclusions of [27]. E.g., for a simple collection experiment limiting currents simulated for the disk match (usually within $0.5 \%$ of error) the predictions of the Levich equation [33], and there is also a good agreement (less than $2 \%$ of error) between simulated and theoretical [3] collection efficiencies.

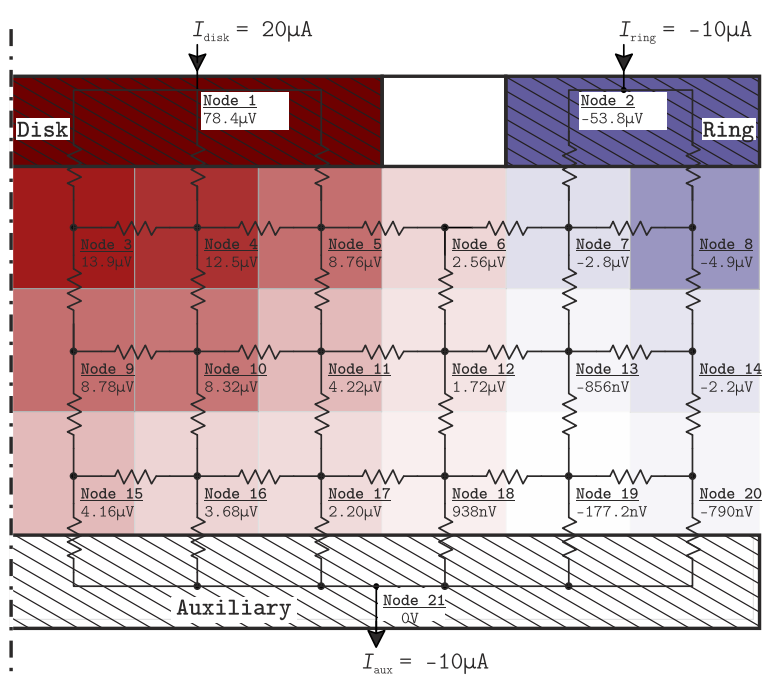

Fig. 2 Interpretation of the simulation grid as a network of electrical resistances. As an example, potential distributions have been calculated for a case when currents of opposite sign enter "Disk" and "Ring". In this illustrative calculation, the resistance values of the circuit have all been set to a value of $1 \Omega$, except for the resistances crossing the "Disk" or "Ring" boundary: these have a value of $10 \Omega$. The intensity of colouring is in accordance with the $\psi$ value for each cell; cold colours stand for negative, warm colours for positive potentials.

\subsection{IR-drop (cross-talk) effects}

In order to implement the simulation of $I R$-drop related cross-talk effects in our model, we interpret the simulation mesh as a network of electrical resistors, like the one shown in Fig. 2.

In case we have a total number of $n \cdot m$ control volumes in the simulation mesh, the "equivalent circuit" of the mesh will contain $n \cdot m+3$ equipotential nodes, due to the three metallic conductors (the disk, the ring and the auxiliary electrode) present in the system. The electric potentials of each node will be ordered in the vector $\psi$ according to the following indexing rule:

- $\boldsymbol{\psi}_{k}$ is the electric potential corresponding to the $k^{\text {th }}$ control volume of the simulation mesh if $1 \leq k \leq$ $n \cdot m$;

- $\boldsymbol{\psi}_{k}$ is the electric potential corresponding to the disk electrode if $k=n \cdot m+1$;

- $\boldsymbol{\psi}_{k}$ is the electric potential corresponding to the ring electrode if $k=n \cdot m+2$;

- $\boldsymbol{\psi}_{k}$ is the electric potential corresponding to the auxiliary electrode if $k=n \cdot m+2$.

Similarly the currents leaving or entering the circuit at each node will be ordered in the vector $\boldsymbol{\imath}$. Since current cannot enter or leave the system, except through the aforementioned electrode metals, $\boldsymbol{\imath}$ will have only three non-zero entries (its last three entries), the sum of which is 0 . 
Regarding the conductances (inverse resistances) present in the equivalent circuit, we note the following:

- Between neighbouring control volumes (with indices $k$ and $\ell$ ) the symmetric $\sigma_{k, \ell}=\sigma_{\ell, k}$ conductance is obtained from the constant bulk conductivity $\kappa$ as

$\sigma_{k, \ell}=\sigma_{\ell, k}=\frac{\kappa A_{k, \ell}}{d_{k, \ell}}$,

where $A_{k, \ell}$ is the bounding area between the two control volumes and $d_{k, \ell}$ is the distance of their center points.

- If a control volume with index $k$ neighbours the disk electrode $(\ell=n \cdot m+1)$, the ring electrode $(\ell=n \cdot m+2)$ or the auxilliary electrode $(\ell=n \cdot m+3)$ then, due to the assumption that all electrode processes are reversible,

$\sigma_{k, \ell}=\sigma_{\ell, k}=\infty$

In practice, usually a very high $(1000 \mathrm{~S})$ value is assumed here instead of infinity.

- If two nodes (with indices $k$ and $\ell$ ) are not neighbours, then

$\sigma_{k, \ell}=\sigma_{\ell, k}=0$.

Based on the above definitions it is possible to construct the Kirchhoff operator K, an $(n m+3) \times(n m+3)$ matrix $^{1}$ as

$\mathrm{K}_{k, \ell}= \begin{cases}-\sigma_{k, \ell} & \text { if } \ell \neq k \\ \sum_{q \neq k} \sigma_{(k, q)} & \text { if } \ell=k\end{cases}$

In order to estimate the ohmic drop affecting the two working electrodes, the matrix-vector representation of Kirchhoff's current-laws may be written as

$\mathrm{K} \boldsymbol{\psi}=\boldsymbol{\imath}$,

and Eqn. (14) has to be solved for $\boldsymbol{\psi}$. Since $\mathrm{K}$ is singular, solving Eqn. (14) is only possible by calculating the generalized (or Moore-Penrose) inverse [35] $\mathrm{K}^{\dagger}$, after which the vector of electric potentials can be obtained as

$\boldsymbol{\psi}=\mathrm{K}^{\dagger} \boldsymbol{l}$.

It is known that instead of using any (slow) iterative methods for computing the Moore-Penrose inverse, $\mathrm{K}^{\dagger}$ can also be calculated [34] directly as

$\mathrm{K}^{\dagger}=\left(\mathrm{K}+\frac{1}{n m+3} \mathrm{~J}\right)^{-1}-\frac{1}{n m+3} \mathrm{~J}$,

\footnotetext{
1 In the context of graph theory, the Kirchhoff matrix is often alternatively called the (weighted) Laplace matrix, see [34].
}

where $J$ is an $(n m+3) \times(n m+3)$ matrix, all the entries of which are equal to 1 . The matrix inversion in Eqn. (16) is still a rather time consuming computation; however, since the resistances in the network remain essentially unchanged during the simulation (this is a result of assuming reversible electrode reactions), it is enough to calculate $\mathrm{K}^{\dagger}$ once, only at the start of the simulations. Using the $\mathrm{K}^{\dagger}$ matrix it is then possible to obtain the $\boldsymbol{\psi}$ vector in each iteration step from Eqn. (15). In Fig. 2 we show a potential field determined by the calculations outlined above, for the case when currents of opposite sign enter the "disk" and "ring" electrodes in a simplified equivalent circuit.

The vector $\boldsymbol{\psi}$ obtained from Eq. (15) is only determined up to an additive constant, which is due to the fact that $\mathrm{K}$ is a positive semi-definite matrix that has exactly one zero eigenvalue with a corresponding element-wise constant eigenvector. The $\delta_{I R \text {, disk }}$ and $\delta_{I R \text {, ring }}$ terms, which - as we will see - are responsible for the appearance of electrical cross-talk must therefore be defined in the form of a difference:

$\delta_{I R, \text { disk }}=\boldsymbol{\psi}_{1}-\boldsymbol{\psi}_{r}$
$\delta_{I R, \text { ring }}=\boldsymbol{\psi}_{2}-\boldsymbol{\psi}_{r}$,

where $r(1 \leq r \leq n m)$ is the index of the control volume chosen as the reference point for voltage measurement. Note that the above model relies on the axial symmetry of the RRDE geometry, thus "reference point" in this context means a "reference annulus-shaped simulation cell". Still, as we will show below, the reference point for voltage measurement is in practice almost equivalent to the position of the tip of a Luggin probe, and can have a very deep impact on the current-voltage characteristics of the two working electrodes.

The simulation algorithm described above was implemented in a software created in the National Instruments LabVIEW development environment. Simulating a $T$ minutes long experiment on a standard platform (PC with 1.6 GHz Dual-Core processor, $4 \mathrm{MByte}$ RAM) takes approximately $6 T$ time for the software.

\section{Experimental}

A PINE AFE7R8 Pt/Pt RRDE was polished by P4000 $\mathrm{SiC}$ paper, rinsed with ultra-pure water (distilled water passed through a Milli-Q system), and then immersed into a solution that contained $10 \mathrm{mmol} \mathrm{dm}^{-3}$ $\mathrm{K}_{3}\left[\mathrm{Fe}(\mathrm{CN})_{6}\right], \quad 10 \mathrm{mmol} \mathrm{dm}^{-3} \mathrm{~K}_{4}\left[\mathrm{Fe}(\mathrm{CN})_{6}\right]$ and $0.5 \mathrm{~mol} \mathrm{dm}^{-3} \mathrm{Na}_{2} \mathrm{SO}_{4}$. The volume of the solution was $\sim 120 \mathrm{~cm}^{3}$. Prior to the measurements the cell was deaerated with an Ar flow. All the used chemicals were 
obtained from Reanal, Hungary and were of purissimum grade; ultra-pure water was used for preparing the solution.

In order to keep the current distribution as homogeneous as possible, some $10 \mathrm{~cm}^{3}$ of $\mathrm{Hg}$ was placed in the cell (before adding the solution). This "mercury lake" was contacted by a Pt needle that was otherwise isolated from the solution, and used as an auxiliary electrode. Saturated calomel electrode (SCE) was used as a reference, and was contacted to the working electrode compartment by the use of a Luggin probe that ended in a fine capillary. The Luggin probe itself was filled with the same solution as the working electrode compartment. The entire cell was movable under the RRDE tip, which allowed us to choose different positions of the Luggin probe with respect to the disk and ring.

A PINE AFRDE5 potentiostat was used in combination with the lab-built measuring system described in [7] for carrying out the experiments.

\section{Results}

In order to demonstrate the effect of $I R$-drop related cross-talk in an RRDE system, simulations and experiments were both carried out. It was assumed in the simulations that the species Red and Ox are, similarly to the measurement, present in equal $\left(10 \mathrm{mmol} \mathrm{dm}^{-3}\right)$ concentrations. An RRDE geometry identical to that of the PINE AFE7R8PtPt tip (used in the experiments) was considered and the parameters listed in Tab. 1 were used in the simulation algorithm. In case of the simulations the standard potential $\left(E^{\ominus}\right)$ of the electrode reaction according to Eqn. (1) was assumed to be $0 \mathrm{~V}$ vs. an "ideal" reference electrode. For the experimental cell, an $E^{\ominus} \approx 175 \mathrm{mV}$ value was determined by measuring the open-circuit potential.

Fig. 3 shows simulated and measured results for a simple collection experiment. In this experiment cyclic voltammograms (sweep rate: $50 \mathrm{mV} \mathrm{s}^{-1}$ ) were recorded at the disk electrode in a $\pm 325 \mathrm{mV}$ wide potential window about $E^{\ominus}$. The rotation rate of the RRDE tip was set to $100 \mathrm{~min}^{-1}$, and the ring potential was fixed at the value of $E^{\ominus}$. The ring current was measured and plotted in Fig.3 as a function of the applied disk potential.

Simulations and experiments were both carried out by selecting three different reference points for the voltage measurements. In case of the experiments the Luggin probe was set to different positions as shown by the photographs of Fig.3. In the simulations different values for the $r$ index in Equations (17.a)-(17.b) were chosen so that the situation would mimic the experimental case. In case 1 the reference point for voltage measurement was on the rotation axis, only $98.75 \mu \mathrm{m}$ below the disk; in case 2 it was placed at the same depth as above, but it was radially displaced to under the ring electrode; in case 3 it was again placed on the axis of rotation, but in a depth of $8.725 \mathrm{~mm}$. Simulations were also carried out in an IR-drop free case 4.

As it can be seen in Fig. 3, there is a fair agreement between calculated and experimentally obtained curves. In a cross-talk free case (Fig. 3(a), case 4) the interpretation of the obtained signals is straightforward. As $E_{\text {disk }}$ is scanned from negative to positive values (and then backwards), the solution layer near the disk electrode gets enriched in the Ox (respectively, Red) species. When the disk potential reaches either very negative or very positive values, there is an almost complete turnover of the redox couple in the vicinity of the disk and a limiting disk current [33] is measured. If this volume of solution then reaches the ring electrode, the ring (as its potential is fixed at $E^{\ominus}$ ) re-sets the original condition of $c_{\mathrm{Ox}}=c_{\mathrm{Red}}$ which yields a negative (respectively, positive) ring current.

The presence of $I R$-drop, as shown by Fig. 3, significantly complicates the interpretation of the measured (simulated) curves. In case 3, when the Luggin probe is kept at a high enough distance from the two working electrodes no cross-talk seems to appear, the current/voltage characteristics of the two working electrodes are, however, disturbed by a "normal $I R$-drop effect" [23].

If on the other hand the Luggin probe is set close to either one of the working electrodes, the current of that electrode gives a significant shift to the reference potential and thus to the effective potential of the other working electrode. This becomes the most obvious in case 1 when the reference point for voltage measurement is set close to the disk which results in ill-behaved ring currents. As the reference potential shifts due to the passing of a high disk current, the effective ring potential does not any more equal $E^{\ominus}$, but - in accordance to the sign of $I_{\text {disk }}-$ it gets slightly more positive or negative than that. This explains why the simulated (and measured) ring curves first show a strong "overshooting" during a disk scan (see the peculiar peaklike features in Fig. 3(a) and Fig. 3(b)) following which they converge to a limiting current that is substantially lower compared to the cross-talk free situation. As it can be seen in Fig. 3(a), the intensity of cross-talk is much lower when the Luggin probe is set close to the ring, although there is a slight difference between the disk voltammograms calculated for cases 2 and 3 .

As it can be seen in Fig. 3 our simulation results are in fair agreement with experimentally obtained curves, although in the multi-physics simulation we ignored 

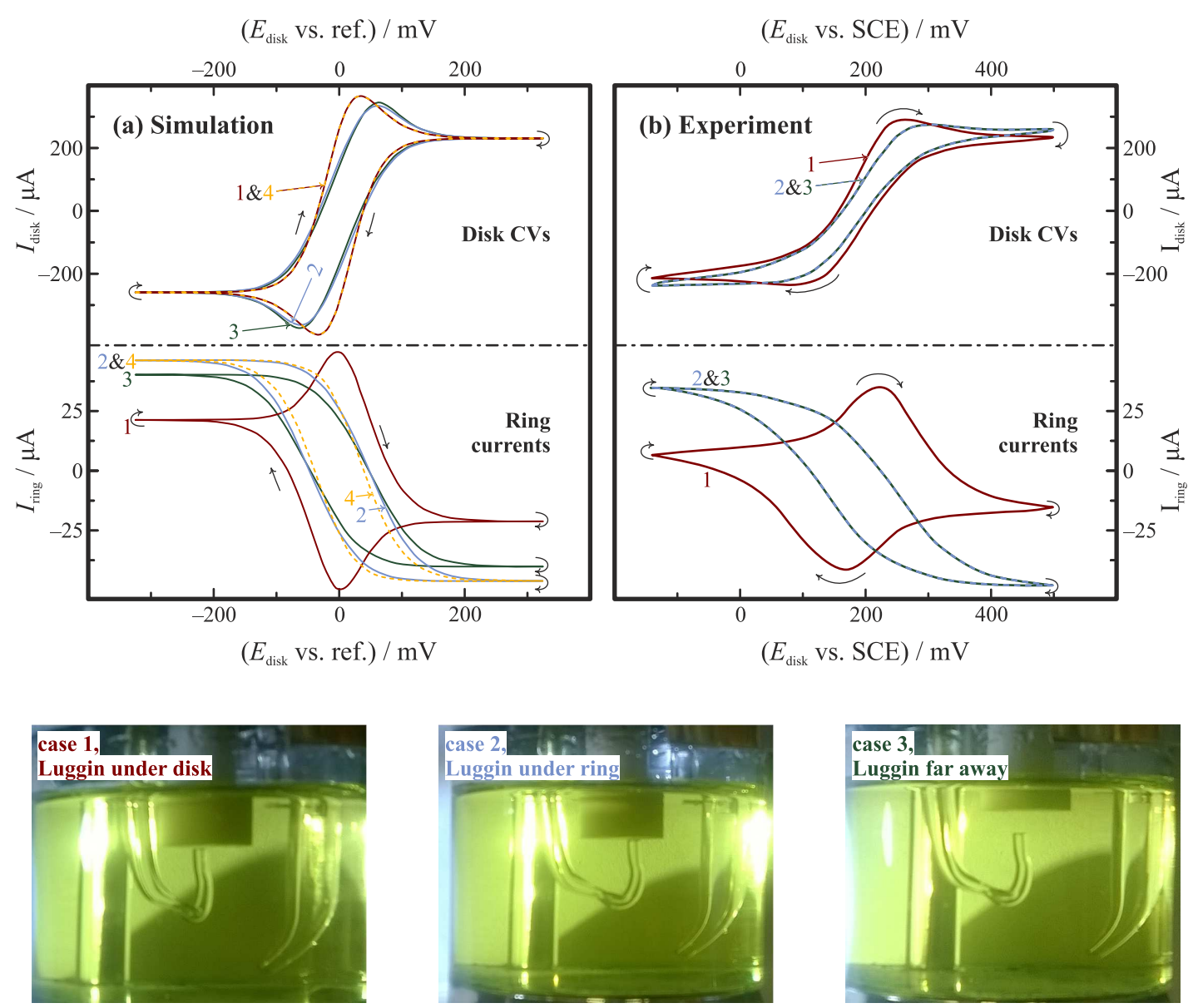

Fig. 3 Simulated (a) and measured (b) disk voltammograms and ring currents recorded in parallel with them. In the simulations the disk is polarized between $-325 \mathrm{mV}$ and $325 \mathrm{mV}$ vs. an "ideal" reference electrode, and a sweep rate of $50 \mathrm{mV} \mathrm{s}^{-1}$ is applied. In the meantime the ring is held at $0 \mathrm{~V}$ vs. the reference electrode (that is, at the value of the open circuit potential). In case of the experiments the open circuit potential is $\sim 175 \mathrm{mV}$ vs. SCE. The disk is polarized at a rate of $50 \mathrm{mV} \mathrm{s}{ }^{-1}$ between limits of $-150 \mathrm{mV}$ and $500 \mathrm{mV}$, and the ring potential is set to $175 \mathrm{mV}$. The applied rotation rate is $100 \mathrm{~min}^{-1}$ for both the simulation and the experiment. In case 4 of the simulation, no $I R$-drop effects are taken into account. In cases 1, 2 and $3 I R$-drop effects are taken into account in the simulations, and the Luggin probe is positioned under the RRDE tip as described in the text. These positions roughly correspond to those used in the experiments, shown by the photographs. (Figure adapted from [28].)

some minor disturbing effects (such as the influence that placing the Luggin probe close to the rotating surface exerts on the hydrodynamic conditions). The modelling of capacitive interfaces is yet to be included to our simulation algorithm, and this will be a subject of a future study.

It should be noted that in a four-electrode system the appearance of cross-talk can be expected whenever the reference point for voltage measurement is set into the high current route of either one of the working electrodes, and it is practically independent from the collection efficiency or the transit time between the working electrodes [3]. In Fig. 3(a) we presented simulation results for a routinely used thin ring-thin gap RRDE configuration, however as shown in Fig. 4, cross-talk can also appear if the gap is much wider and the ring is also of a considerable surface area.

\section{Discussion}

Electrochemistry textbooks, when introducing the concept of IR-drop in a standard three-electrode cell often suggest that its disturbing effect can largely be eliminated if "the reference electrode is designed for very close placement to the working electrode by use of a fine tip called a Luggin-Haber capillary" [3]. We have seen, however, that following the very same strategy in a four-electrode cell can lead to the appearance of heavy cross-talk and can seriously complicate the interpretation of measured data.

The fact that a fair agreement can be found between simulations and measurements implies that the model presented in Section 2 of this paper describes well both the cross-talk and the "normal IR-drop" effects. In what follows we shall use the results of digi- 


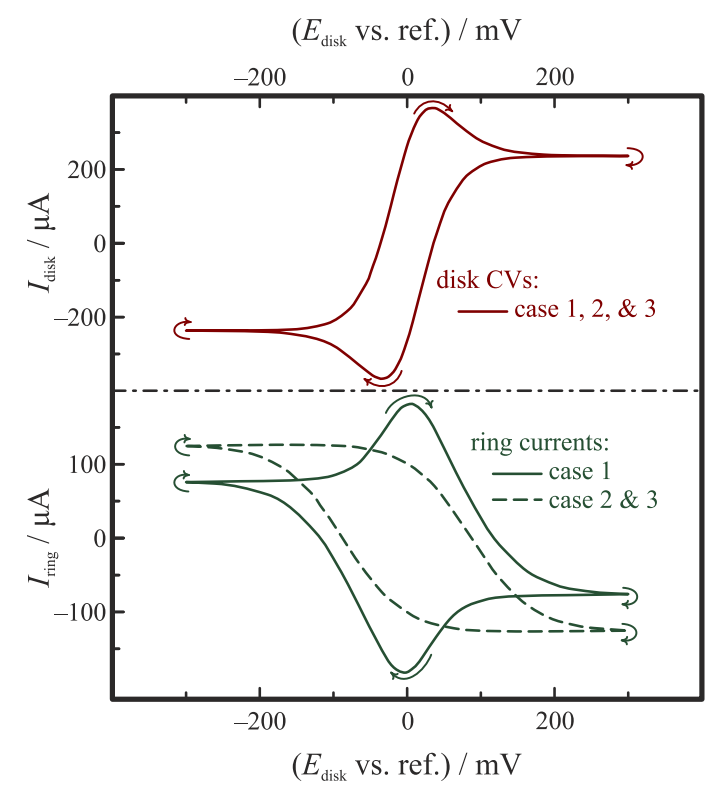

Fig. 4 Curves similar to those shown in Fig. 3(a), now simulated for an RRDE with bigger gap size $(1 \mathrm{~mm})$ and a thicker ring $(1 \mathrm{~mm})$. The placement of the reference electrode in cases 1-3 matches that of Fig.3; case 4 corresponds to an $I R$-drop free situation.

tal simulations in order to define some rules of thumb that can help experimentalists working with RRDE (or other GC) systems in dealing with the effects of crosstalk.

\subsection{Diagnostic criteria}

Whenever a four-electrode system is used, it is always advisable to rule out the presence of cross-talk. This can be done, e.g., by running experiments using differently positioned Luggin probes, and then comparing the measured results. If no differences are detected, this is a good indication of a cross-talk free system. A large advantage of using an RRDE is the possibility to conduct experiments also in the absence of rotation. Strong disk/ring interactions measured on a stagnant RRDE indicate the presence of cross-talk (Fig. 5), viz. in the absence of convection no true collection should occur.

\subsection{Eliminating cross-talk by the use of two references}

Probably the most straightforward way of eliminating $I R$-drop effects when two working electrodes are used in the same cell is to upgrade the cell from a fourelectrode to a five-electrode system. That is, a second reference electrode should be introduced and an appropriate bi-potentiostatic control circuit that allows

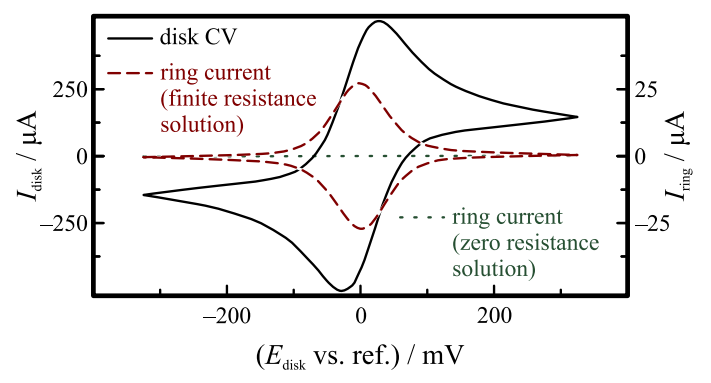

Fig. 5 In a cross-talk free case (i.e., in a solution of zero resistance) the ring signal on an unrotated RRDE should be essentially zero, as the ring potential is fixed at $E^{\ominus}$, and in the absence of rotation the concentration changes near the disk electrode have very little effect on the ring. The appearance of diskinduced ring currents on a stagnant RRDE indicates cross-talk; this can be explained by a shifting of the effective $E_{\text {ring }}$ value by strong disk currents.

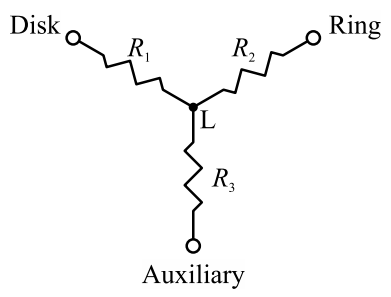

Fig. 6 Simplified equivalent circuit of the RRDE system. L shows an optimum (cross-talk free) position for the Luggin capillary.

the connection of this second reference should be applied. When the electrode potentials of each working electrodes are measured with respect to their own dedicated reference, by the use of two appropriately positioned Luggin capillaries - each oriented to a respective working electrode - it may become possible to rule out cross-talk effects almost entirely. Nevertheless the above strategy is, due to instrumental difficulties, rarely applied, and most practical generator/collector configurations are built as four-electrode systems.

\subsection{Eliminating cross-talk by Luggin probe positioning}

In a large enough four-electrode cell (like that of the RRDE) one may often find a suitable Luggin probe position using which cross-talk effects can be minimized. ${ }^{2}$ Theoretically, this is possible by measuring the resistances (e.g., by two-points impedance measurements) between the disk and the ring ( $\left.R_{\text {disk to ring }}\right)$, between the disk and the auxiliary electrode $\left(R_{\text {disk to aux }}\right)$

\footnotetext{
2 When using other four-electrode configurations requiring miniaturized cells like the SECM, conducting probe AFM or electrochemical STM, such an effort may still be in vain.
} 
and between the ring and the auxiliary electrode ( $\left.R_{\text {ring to aux }}\right)$. Using these resistances it is possible to construct an equivalent circuit of the cell [20], depicted in Fig. 6, where the given resistances can be calculated as

$R_{1}=\frac{1}{2}\left(R_{\text {disk to aux }}+R_{\text {disk to ring }}-R_{\text {ring to aux }}\right)$,

$R_{2}=\frac{1}{2}\left(-R_{\text {disk to aux }}+R_{\text {disk to ring }}+R_{\text {ring to aux }}\right)$,

$R_{3}=\frac{1}{2}\left(R_{\text {disk to aux }}-R_{\text {disk to ring }}+R_{\text {ring to aux }}\right)$.

Cross-talk effects in the system shown in Fig. 6 can be eliminated by placing the Luggin probe to the position marked with L; that is, to the junction of the three resistors. With respect to this approach some remarks are however due. i.) Strictly speaking, the resistances in Eqs. (18.a)-(18.c) include not only solution resistances, but also the interfacial resistances of the electrodes. As the latter may change in the course of the experiment (e.g., as a function of the electrode potentials applied), the optimum Luggin position may also shift within the cell. ii.) Using the optimum Luggin probe position, although cross-talk effects may be eliminated, a ,normal $I R$-drop” [23] still affects both working electrodes $\left(\delta_{I R, \text { disk }}=I_{\text {disk }} R_{1}\right.$ and $\left.\delta_{I R, \text { ring }}=I_{\text {ring }} R_{2}\right)$.

\subsection{Correction of curves measured in the presence of} cross-talk

If cross-talk effects in an RRDE system cannot be eliminated experimentally, it may still be expedient to look for methods of correcting the measured data.

Consider as an example a collection experiment when both the disk and the ring electrodes are under potentiodynamic control. While the disk is slowly scanned from positive to negative values, we record CVs at a high scan rate on the ring electrode. This method, as shown in $[6,22]$ can yield a useful threedimensional map showing at which disk potentials some electroactive products are formed, and at which ring potentials they can be detected. The $3 \mathrm{D}$ map is created by subtracting from the measured ring currents a "reference ring voltammogram" (recorded at an appropriate $E_{\text {disk }}$ value where no collection is assumed to occur), and then plotting the thus obtained $\Delta I_{\text {ring }}$ values as a bivariate function of the disk and ring potentials.

By the means of simulations we created such a 3D map for the simple redox system studied in this paper. For a cross-talk free case, results are shown in Fig. 7: the 3D map shows that the collection of excess amounts of redox-active species shifts the ring current
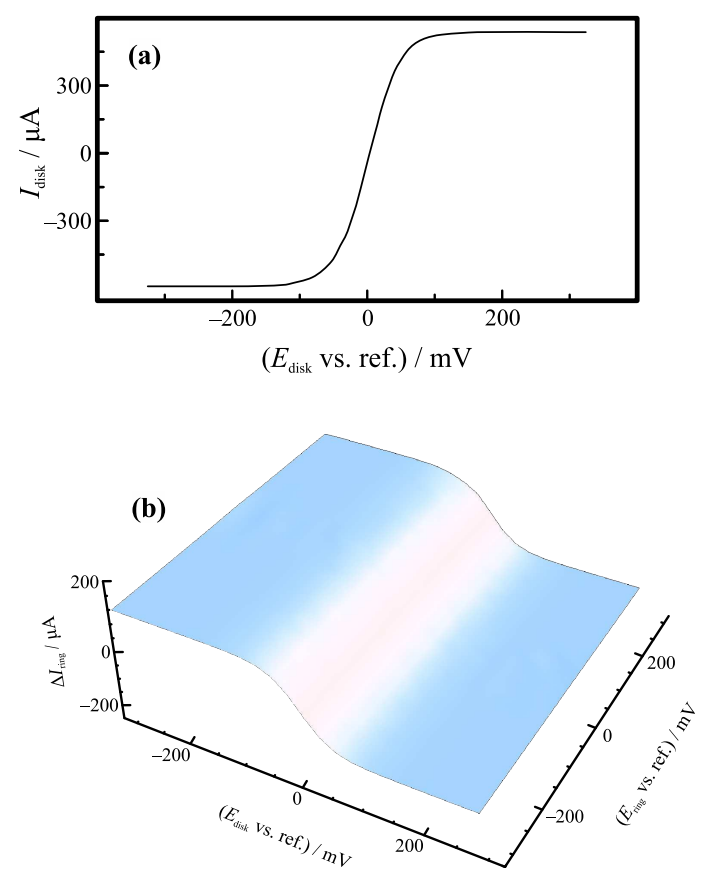

Fig. 7 A disk voltammogram (a) and a 3D map (b) [6, 22] simulated for the simple redox system under study. At a rotation rate of $500 \mathrm{~min}^{-1}$, the disk was slowly scanned (sweep rate: $\left.1 \mathrm{mV} \mathrm{s}^{-1}\right)$ from very positive $(325 \mathrm{mV})$ to very negative $(-325 \mathrm{mV})$ values and thus the voltammogram shown in (a) was obtained. Simultaneously, the ring potential was scanned between the same vertices at a high sweep rate $\left(100 \mathrm{mV} \mathrm{s}^{-1}\right)$. Changes of the ring currents (measured during positively going ring sweeps) were plotted as a function of the disk and ring potentials and thus the surface shown in (b) was obtained. A ring sweep taken at $E_{\text {disk }}=0 \mathrm{~V}$ was used as a reference for calculating the plotted current differences.

over the whole $E_{\text {ring }}$ range studied. This shift, as shown by Fig. 7, has a sign opposite than that of the disk current.

As opposed to the $I R$-drop free case the threedimensional map recorded in a system of finite resistance (with the reference point for voltage measurements positioned close to the disk) shows a significant cross-talk related artefact (Fig. 8(a)). This artefact may however be eliminated by applying a point-wise correction to each $E_{\text {ring }}$ values of the dataset in the form of

$E_{\text {ring, corr }}=E_{\text {ring }}-\delta_{I R, \text { ring }}$.

As the reference point for voltage measurement was in this case positioned close to the disk,

$\delta_{I R, \text { ring }}=R_{2} I_{\text {ring }}-R_{1} I_{\text {disk }}$,

using the notations of Fig. 6. That is, by determining the values of $R_{\text {disk to ring }}, R_{\text {disk to aux }}$ and $R_{\text {ring to aux }}$ in an RRDE systems, curves measured in a heavily crosstalk affected case can be corrected post-experimentally. 

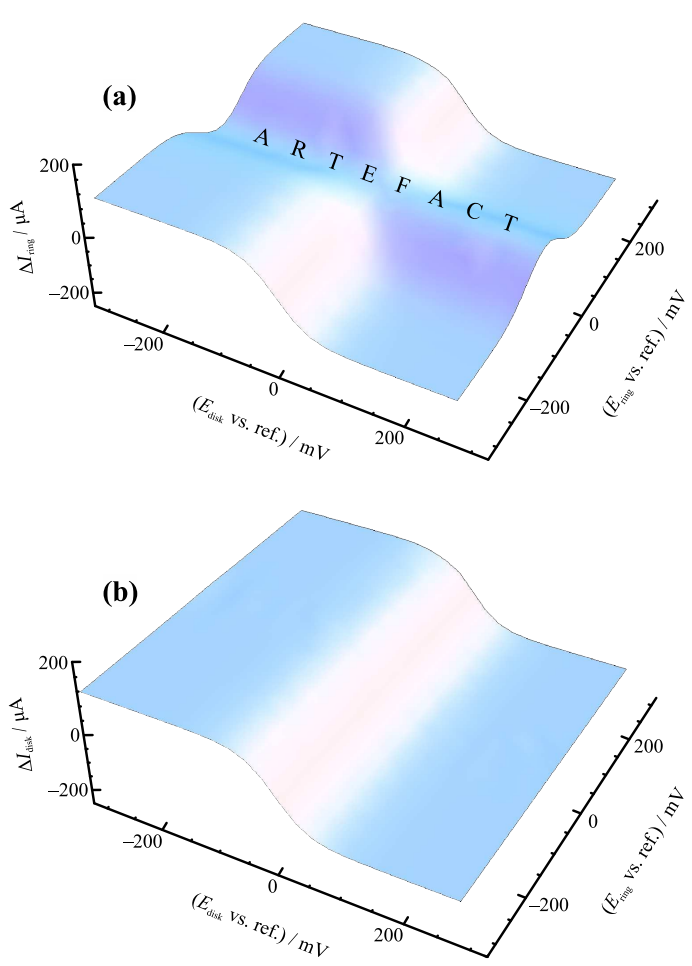

Fig. 8 A 3D map simulated for a system of finite resistance when the reference point for voltage measurement placed close to the disk (a) shows an intense cross-talk related artefact. By using the method described in the text, this artefact may be corrected for and the thus obtained surface (b) is similar to the one measured for an $I R$-drop free case (cf. to Fig. 7(b)).

\section{Conclusion}

We presented a digital simulation model that can not only describe the electrochemical generation and collection of redox-active species in four-electrode configurations, but - by solving the Kirchhoff equations over the simulation mesh - it can also account for the electrical cross-talk of the two working electrodes. By simulating a simple collection experiment with a rotating ring-disk electrode, the model predictions were explained and experimentally verified.

In accordance with previous studies [19-23] we found that electrical cross-talk in four-electrode systems may arise due to the shared current routes of the working electrodes and that the intensity of the crosstalk depends heavily on the position of the reference electrode. While in Refs. [19-22] cross-talk was explained and treated on the basis of a simplified analogy to star-shaped equivalent circuits, and thus these works could model only the electrical (but not the chemical) interactions between the two working electrodes, the model described in this paper can account for both types of interactions. A novelty of the presented approach (over [23], for example) is that it does not ne- cessitate the application of galvanostatic control on both working electrodes. It should be emphasized that cross-talk may have a larger disturbing effect on the current $v s$. potential characteristics of the two working electrodes in case transient techniques are applied, and thus stronger currents are passing the electrodes under study.

The electrochemical system targeted here was the rotating ring-disk electrode (RRDE); the paper described several strategies to reveal/eliminate/correct for the effects of cross-talk. In an RRDE system suitable Luggin probe positions (that are out of any high current routes) can often be found in order to reduce crosstalk effects to an almost negligible intensity. This, however, may not the case when we use four-electrode arrangements in very small cells, where the position of the reference electrode is not so freely chosen. Thus the results of SECM experiments (and in some cases also measurements made in STM or conducting-probe AFM configurations) may also get affected by electrical cross-talk, in a manner similar to what was presented here for RRDEs.

Acknowledgements Some of the chemicals used in this study were donated by G. Inzelt (ferrocyanides) and by T. Pajkossy (mercury), for which the authors express their gratitude. The help of T. Pajkossy in taking the photographs shown in the paper is also highly appreciated. The donation of data acquisition cards used for the experiments is gratefully acknowledged to National Instruments Hungary Ltd., Debrecen.

This research was supported by the European Union and the State of Hungary, co-financed by the European Social Fund in the framework of TÁMOP 4.2.4.A/1-11-1-2012-0001 National Excellence Program. S. Vesztergom thanks the Scientific Exchange Programme NMS ${ }^{\text {ch }}$ (SciEx 13.060) for funding this work. G.G. Láng gratefully acknowledges support from the Hungarian Scientific Research Fund (OTKA-K109036). P. Broekmann acknowledges financial support by the Swiss National Science Foundation (SNSF 200020_149224/1).

\section{References}

1. Frumkin AN, Nekrasov LN, Levich VG, Ivanov YB (1959) J Electroanal Chem 1:84-89

2. Barnes EO, Lewis GEM, Dale SEC, Marken F, Compton RG (2012) Analyst 137:1068-1081

3. Bard AJ, Faulkner LR (2001) Electrochemical methods: Fundamentals and applications, 2nd edn. Wiley, New York

4. Bard AJ, Mirkin MV (eds) (2012) Scanning Electrochemical Microscopy, 2nd edn. CRC Press, Boca Raton FL

5. Vesztergom S, Ujvári M, Láng GG (2011) Electrochem Commun 13:378-381 
6. Vesztergom S, Ujvári M, Láng GG (2012) Electrochem Commun 19:1-4

7. Vesztergom S, Láng GG (2013) Instrum Sci Technol 41:82-95

8. Vesztergom S, Ujvári M, Láng G (2013) Dual dynamic voltammetry with rotating ring-disk electrodes. In: Saito Y, Kikuchi T (eds) Voltammetry: Theory, Types and Applications, Nova Science Publishers, New York

9. Shen Y, Träuble M, Wittstock G (2008) Anal Chem 80:750-759

10. Eckhard K, Chen X, Turcu F, Schuhmann W (2006) Phys Chem Chem Phys 8:5359-5365

11. Schwager P, Fenske D, Wittstock G (2015) J Electroanal Chem 740:82-87

12. Schwager P, Dongmo S, Fenske D, Wittstock G (2016) Phys Chem Chem Phys 18:10774-10780

13. Albery WJ, Compton RG, Hillman AR (1978) Trans Faraday Soc 74:1007-1019

14. Albery WJ, Hillman AR (1979) Trans Faraday Soc 75:1623-1634

15. Benzekri N, Keddam M, Takenouti H (1989) Electrochim Acta 34:1159-1166

16. Kovács N, Ujvári M, Láng GG, Broekmann P, Vesztergom S (2015) Instrum Sci Technol 43:633648

17. Trinh D, Keddam M, Novoa XR, Vivier V (2011) ChemPhysChem 12:2167-2176

18. Trinh D, Keddam M, Novoa XR, Vivier V (2011) ChemPhysChem 12:2177-2183

19. Trinh D, Maisonhaute E, Vivier V (2012) Electrochem Commun 16:49-52

20. Shabrang M, Bruckenstein S (1974) J Electrochem Soc 121:1439-1444

21. Gabrielli C, Keddam M, Takenouti H (1972) J Chim Phys Phys -Chim Biol 69:737-740

22. Vesztergom S, Ujvári M, Láng GG (2013) Electrochim Acta 110:49-55

23. Dörfel C, Rahner D, Forker W (1980) J Electroanal Chem 107:257-270

24. Prater KB, Bard AJ (1970) J Electrochem Soc 117:207-213

25. Feldberg SW, Bowers ML, Anson F (1986) J Electroanal Chem 215:11-28

26. Farkas J, Kiss L, Fóthi Á (1980) Acta Chim Acad Sci Hung 104:405-415

27. Vesztergom S, Barankai N, Kovács N, Ujvári M, Wandlowski T, Láng GG (2014) Acta Chim Slov 61:223-232

28. Vesztergom S, Barankai N, Kovács N, Ujvári M, Broekmann P, Siegenthaler H, Láng GG (2016) Electrochem Commun 68:54-58
29. Riddiford AC (1966) Rotating disc systems. In: Delahay P, Tobias CW (eds) Advances in electrochemistry and electrochemical engineering, vol 4, Interscience, New York

30. Kármán T (1921) Z Angew Math Mech 1:233-252

31. Cochran WG (1934) Math Proc Cambridge Phil Soc 30:365-375

32. Nelder JA, Mead R (1965) Comput J 7:308-313

33. Levich VG (1962) Physicochemical Hydrodynamics. Prentice Hall, Englewood Cliffs, NJ

34. Gutman I, Xiao W (2004) Bull Acad Serbe Sci Arts 129:15-23

35. Ben-Israel A, Greville TNE (2003) Generalized Inverses. Springer, Heidelberg 